\title{
EINE ÄTHIOPISCHE MAGISCHE ROLLE UND IHRE ILLUSTRATIONEN*
}

\author{
Einleitung
}

Die äthiopische Magie samt ihren Utensilien - Zauberrollen, Amulette, Zauberbücher - fußt auf einer Tradition, die gemeinsame Züge mit ähnlichen Vorstellungen und Gegenständen aus dem Alten Orient und dem Mittelmeerraum aufweist. Hier sollen kurz einige Merkmale der magischen Literatur Äthiopiens bzw. der Zauberrollen genannt werden. Im 4. Jh. n. Chr. nahm der König 'Ezānā das Christentum an. Die allmähliche Christianisierung des damaligen Reichs von Aksum ging mit der Übernahme von hellenistisch-synkretistischem Gedankengut der Spätantike einher. Die Tatsache, dass nur drei Jahrhunderte später die Verbreitung des Islam die Verbindungen des aksumitischen Reichs mit der übrigen christlichen Welt erschwerte, führte zur Entfaltung eines Christentums semitischer Prägung, das viele archaische Züge beibehalten hat. Da im Laufe der Zeit Äthiopien eine Randzone der christlichen Welt wurde, die durch Konservatismus gekennzeichnet ist, konnten sich einige Charakteristika über Jahrhunderte behaupten. Die jüdischen Engel- und Dämonenlehren sind in der äthiopischen Literatur gut vertreten, obgleich sie durch eigene Beiträge erweitert wurden.

Der Untergang des aksumitischen Reichs Ende des 8. Jh. n. Chr. bedeutete keineswegs das Ende dieser christlich geprägten Kultur. Nach einigen - in Bezug auf unsere Kenntnis davon „dunklen“ Jahrhunderten begann im 13. Jh. n. Chr. ein Prozess, der bis ins 20. Jh. andauerte: die allmähliche Eroberung und teilweise Christianisierung des „heidnischen“, kuschitischen Südens, eine Art translatio imperii von Norden nach Süden. Diese Entwicklung wird u. a. an der nach Süden gerichteten Verlagerung der Reichshauptstadt deutlich: Aksum, Roha, Gondar, Ankobar, Addis Abeba. Dieser langwierige Prozess, den man als Akkulturation bezeichnen kann, bewirkte, dass Kuschitisches gleichermaßen in das Christlich-Semitische integriert wurde. Dazu kam ein anderer wichtiger Aspekt. Neben dem offiziellen Christentum, das institutionalisiert und fest geregelt war und im Laufe der Zeit entsprechend den jeweiligen historischen Verhältnissen die äthiopische Gesellschaft prägte, konnte sich auf der Basis des vorchristlichen Substrats eine eigenständige Volksfrömmigkeit etablieren - eine Synthese, in der jüdische, vorchristlich-afrikanische, christliche und islamische Elemente nebeneinander existieren und ineinander übergehen. Gerade in diesem Milieu behaupteten und bewährten sich Magie und Zauberei über lange Zeit.

Eine der bekanntesten Ausdrucksformen äthiopischen magischen Gedankenguts stellen die sog. Zauberrollen dar. Das Herstellen von Zauberrollen oder Amuletten ${ }^{1}$, die man auf Amharisch $k \partial t a b^{2}$ oder genauer yä-bəranna kətab (,,auf Leder Geschriebenes“) nennt, gehört zu den

\footnotetext{
* In memoriam Rosaria Golsch (emeritierte Äbtissin von Marienkron).

${ }^{1}$ Sie werden von vielen Völkern Äthiopiens unabhängig von ihrer Konfession getragen, vgl. die jeweiligen Bezeichnungen bei J. S. Trimingham, Islam in Ethiopia. London 1965, 263 Anm. 1.

${ }^{2}$, ,Ketab literally means amulets kept around the neck“, TseHAi Berhane Selassie, An Ethiopian Medical TextBook written by Gerazmač Gäbräwäld Arägahäñ Däga Damot. Journal of Ethiopian Studies 9 (1971) 142 Anm. 40. Es handelt sich um ein Lehnwort aus dem Arabischen: kitāb bedeutet „Schreiben, Schriftstück; Brief; Urkunde; Kontrakt; Buch“. Das arabische kitāba tun bedeutet unter anderem auch ,geschriebenes Amulett, Zauberspruch“, H. WEHR, Arabisches Wörterbuch für die Schriftsprache der Gegenwart. Wiesbaden ${ }^{5} 1985,1085$.
} 
wichtigsten Tätigkeiten der äthiopischen Dabtarā. Als Mitglieder einer Klasse niederer, ungeweihter Geistlicher gehören die Dabtarā nicht der Hierarchie der äthiopischen Kirche an. Was das kirchliche Leben betrifft, haben sie als Laien jedoch eine wichtige Funktion in ihren Gemeinden. So sind sie nicht nur ausgezeichnete Kenner der liturgischen Vorschriften und als solche des Altäthiopischen $\left(\mathrm{G} \partial^{6} \partial \mathrm{z}\right)^{3}$ mächtig, sondern haben auch eine musikalische Ausbildung genossen, die es ihnen erlaubt, die hochkomplizierten liturgischen Gesänge zu interpretieren ${ }^{4}$. Darüber hinaus sind sie durch ihre Ausbildung imstande, zum Zwecke des Gottesdienstes bei der Bevölkerung sehr beliebte Dichtungen zu komponieren und vorzutragen ${ }^{5}$. In den Dorfschulen übernehmen sie die Rolle des Lehrers für die Bauernkinder. Ihre Aufgabe in der äthiopischen Gesellschaft wird aber nicht nur durch ihre Stellung innerhalb des offiziellen Kults bestimmt. Sie haben zugleich einen festen Platz in der äthiopischen Volksreligion, wo sie als Zauberspezialisten und Heiler auftreten.

Formal gesehen handelt es sich bei den Zauberrollen um Pergamentstreifen von etwa 10 bis 20 Zentimeter Breite und ein bis zwei Meter Länge. Je nach der Größe der Tierhaut bestehen sie aus zwei bis vier Einzelstücken, die zusammengenäht und in die Breite beschrieben und bemalt sind $^{6}$. Um sie lesen zu können, entrollt man sie, während das Gelesene wieder eingerollt wird. Zusammengerollt werden sie in einem Lederetui aufbewahrt und um den Hals oder am Körper getragen. Der Text der Zauberrollen wird meist in Altäthiopisch geschrieben, wenn auch mitunter in einem lexikalisch und syntaktisch mangelhaften, vom Amharischen beeinflussten Gə`əz. Es gibt auch Zauberrollen neueren Datums in amharischer Sprache. Die meisten Zauberrollen sind ein Mixtum compositum von Texten verschiedenen Ursprungs, bei denen die Zugehörigkeit zu der einen oder anderen der Textgattungen keine Rolle spielt. Diese heterogene Zusammenstellung von magisch-medizinischen Erfahrungen und christlichem Gedankengut wird allein von der Vertrautheit des Dabtarā mit der Gə‘əวz-Literatur und von den Wünschen und der Notlage (Krankheit, Bedrohung usw.) des Klienten bestimmt. Das soziale Umfeld, in dem diese magischen Rollen in Gebrauch waren bzw. noch sind, ist das der bäuerlichen Gesellschaft des äthiopischen Hochplateaus. Die Kundschaft besteht mehrheitlich aus Frauen, wie die Namen der Rollenbesitzer zeigen.

Außer den Texten enthalten die Zauberrollen auch Bilder. Die meisten Rollen beginnen und enden mit einem Bild. Zudem sind gewöhnlich noch ein oder zwei Bilder in die Texte eingefügt. Eine genaue Datierung der Rollen ist nicht möglich. Die Namen der Besitzer, für die sie geschrieben sind, sagen nichts über deren Lebensdaten aus. Die Schreiber der Rollen bleiben anonym. Die meisten Rollen stammen aus den letzten 300 Jahren, also aus einer Zeit, in der sich die Schriftzeichen wenig verändert haben. Eine Datierung ist daher kaum anders möglich als anhand stilistischer Merkmale der Bilder.

\footnotetext{
${ }^{3}$ Das Altäthiopische (Eigenbezeichnung Gə‘əz) ist die semitische Sprache, die in der Antike im Norden Äthiopiens gesprochen wurde. Obwohl es ab $1000 \mathrm{n}$. Chr. nicht mehr gesprochen wurde, blieb es bis in das 19. Jh. hinein Verwaltungssprache und Literatursprache und wurde erst allmählich vom Amharischen verdrängt. Als Liturgiesprache wird es bis heute von der Äthiopischen und Eritreischen Orthodoxen Kirche gebraucht.

${ }^{4}$ B. Velat, Chantres, Poètes, Professeurs: Les Dabtara éthiopiens. Les Cahiers Coptes 5 (1954) 21-29, bes. 22ff.; K. Kaufman Shelemay, The Musician and Transmission of Religious Tradition: The Multiple Roles of the Ethiopian Däbtära. Journal of Religion in Africa 22 (1992) 242-260, bes. 246ff. und Dies., Zēmā: A Concept of Sacred Music in Ethiopia. The World of Music 24/3 (1982) 52-67.

${ }^{5}$ B. Velat, Chantres, Poètes, Professeurs: Les Dabtara éthiopiens, 24.

${ }^{6}$ Über die Herstellung von Zauberrollen siehe J. MERCIER, Art that heals: The Image as Medicine in Ethiopia. München - New York 1997, 46.
} 


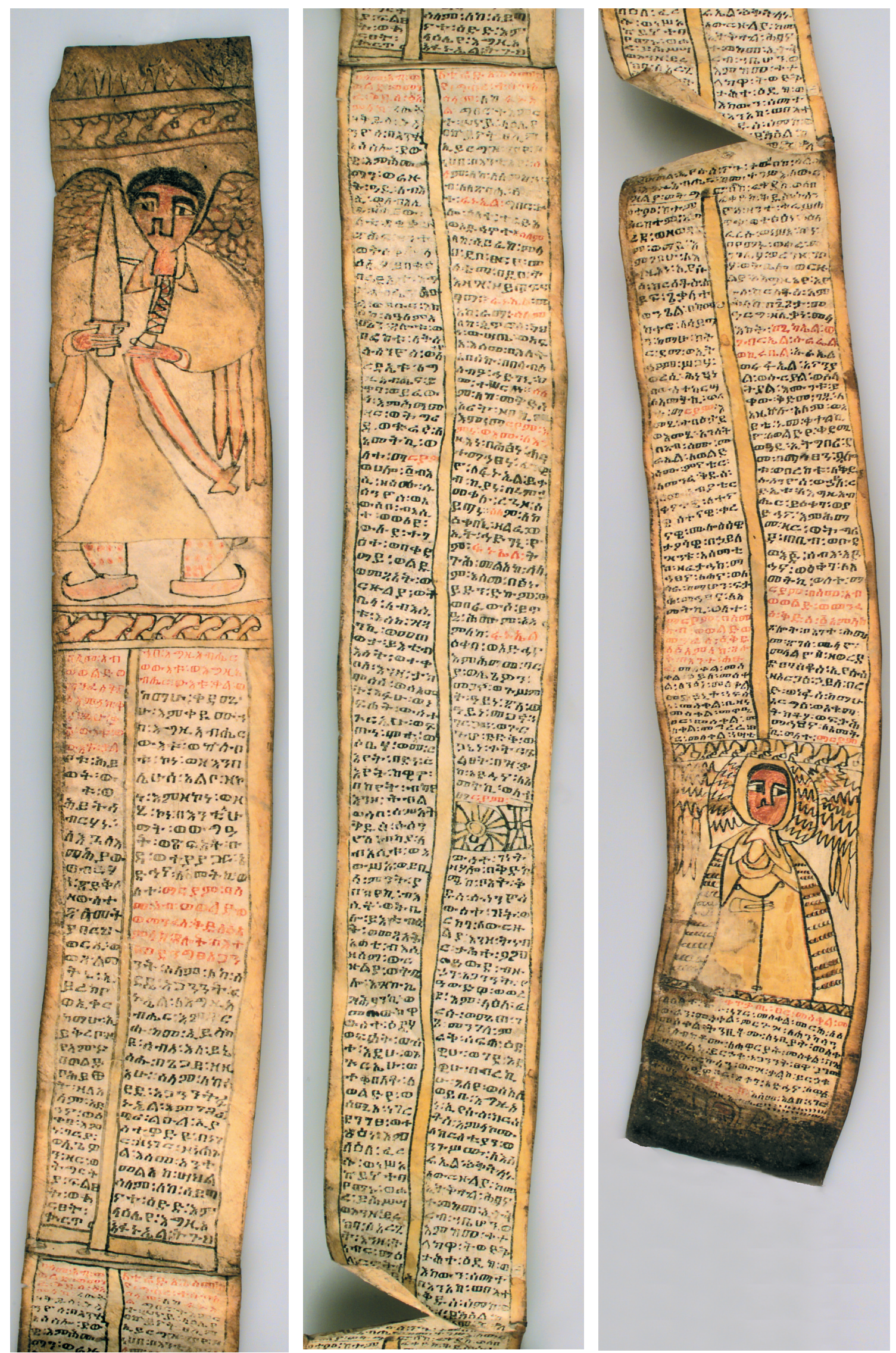

Abb. 1-3 (Foto: Institut für Klassische Archäologie der Universität Wien, E. HÜTTER) 
Beschreibung der Zauberrolle

Über die Herkunft der Rolle weiß man nur, dass sie aus Äthiopien stammt. Der Name des Besitzers - wie in den meisten Fällen eine Frau - Walatta Māryām (Tochter Mariae) wird an mehreren Stellen des magischen Texts erwähnt. Er ist wie die Einleitungsformel der Gebete mit roter Tinte geschrieben.

Die Breite der Rolle beträgt durchschnittlich $12 \mathrm{~cm}$, die Länge circa $197 \mathrm{~cm}$. Die Rolle besteht aus drei Stücken, die durch Lederriemen miteinander verbunden sind: 1 . Stück: ca. $68 \mathrm{~cm}$, 2. Stück: ca. $70 \mathrm{~cm}, 3$. Stück: ca. $59 \mathrm{~cm}$. An den beiden Nahtstellen überlappen sich die Stücke. Die Lederriemen, die das zweite und dritte Stück miteinander verbinden, sind zum Teil zerrissen. Die Rolle ist aus Pergament, das an mehreren Stellen angedunkelt ist.

Der Text der Rolle läuft meist über zwei Spalten von unterschiedlicher Breite, circa 4,5 bzw. 5,5 cm. Der untere Teil der Rolle ist einspaltig geschrieben.

Auf der Rolle befinden sich vier Zeichnungen: ein schützender Engel am Anfang, ein kleines Zeichen, das den Text der zweiten Spalte unterbricht, wiederum ein Engel im unteren Bereich, als Abschluss des zweispaltig geschriebenen Textteils, und am Ende der Rolle eine Zeichnung, die wegen des stark angedunkelten Pergaments kaum mehr erkennbar ist.

Die Schrift ist dick und grob und an einigen Stellen wegen der Zerknitterung des Pergaments schwer lesbar.

Die Sprache der Zauberrolle ist Altäthiopisch. Die Verwendung amharischer Schriftzeichen beschränkt sich auf die Dämonen- und Krankheitsnamen. Fehlerhafte Morphologie und syntaktische Unsicherheiten verraten einen Dabtarā mit geringen Gə‘əz-Kenntnissen.

Datierung: 20. Jahrhundert.

\section{Übersetzung der magischen Gebete}

Die Übersetzung versucht den Originaltext möglichst genau wiederzugeben ${ }^{7}$. In dem Fußnotenkommentar werden wichtige Begriffe der äthiopischen Magie erläutert. Folgende Abkürzungen und Zeichen werden in der Übersetzung verwendet:

(........) unleserliche bzw. unklare Stelle im äthiopischen Text

[ ] Ergänzung dem Sinn nach

[?] Auffälligkeiten des äthiopischen Texts

(Z. 1-7 linke Sp. / Z. 1-11 rechte Sp.)

Im Namen des Vaters und des Sohnes und des Heiligen Geistes, des einen Gottes.

Am Anfang war das Wort ${ }^{8}$. Und dieses Wort war bei Gott. Und Gott war das Wort. Und so war es am Anfang, von Anfang an bei Gott. Und alles ist durch es geworden, aber ohne es ist nichts geworden, welches geworden ist.

(Z. 8-41 linke Sp. / Z. 12-16 rechte Sp.)

Durch es ist das Leben. Das Leben aber ist das Licht des Menschen. Das Licht der Gerechtigkeit ist es, welches in der Finsternis leuchtet und scheint, die Finsternis aber ergreift den nicht und nähert sich nicht demjenigen, welcher an den Sohn glaubt. Er hat das Leben, welches ewig ist.

\footnotetext{
${ }^{7}$ Die Wiedergabe äthiopischer Begriffe richtet sich in den meisten Fällen nach der Transliteration des Altäthiopischen.

${ }^{8}$ Der Prolog des Johannesevangeliums wird häufig magisch gebraucht, s. B. BuRTEA, Zwei äthiopische Zauberrollen (Semitica et Semitohamitica Berolinensia 1). Aachen 2001, 56f.
} 
Schütze sie und rette sie von bāryāa und legewon ${ }^{10}$, zār ${ }^{11}$ und təgrətya $\bar{a}^{12}$, fəlsat ${ }^{13}$, q $^{\text {wortəmă }} t^{14}$, wəg 'ât $t^{15}$ und səf' $a t^{16}$, budâ ${ }^{17}$ und tayāyāž ${ }^{18}$, rette sie, deine Magd, Walatta Māryām.

\section{(Z. 16-81 rechte Sp.)}

Im Namen des Vaters und des Sohnes und des Heiligen Geistes, des einen Gottes.

Gebet betreffend das Erschrecken der Dämonen ${ }^{19}$.

Heil dir, Vertreiber der Dämonen, Fānu'el, aus Gottes Tempel,

damit sie nicht anklagen die Menschen, die

bereuen ein jeder seine Sünde.

${ }^{9}$ Bāryā ist eine der verbreitetsten Dämonengestalten der äthiopischen Zauberliteratur. Es hat auch andere Bedeutungen: nach I. GuIDI, Vocabolario amarico-italiano. Rom 1901, Sp. 327 „schiavo, schiava“, bezeichnet bāryā auch den Menschen, der dem Willen der Dämonen unterworfen ist. Der Anführer dieser Dämonen ist legewon (vgl. Mk 5, 9; Lk 8, 30), was sein häufiges Vorkommen in den Zauberrollen neben bāryā erklären würde. Des Weiteren ist bāryā die Bezeichnung eines schwarzafrikanischen Volkes - heutzutage Nārā genannt - in Eritrea, das wegen seiner von den Christen Äthiopiens als Orgien gedeuteten Besessenheitskulte verrufen war. Die meisten Forscher leiten daraus ab, dass bāryā der Name für Epilepsie sein soll. Vgl. B. BuRTEA, Zwei äthiopische Zauberrollen, 51.

${ }^{10}$ Es handelt sich dabei um eine Übernahme aus der neutestamentlichen Dämonologie (Mk 5, 9. 15; Lk 8, 30). In der äthiopischen Zauberliteratur ist legewon sehr häufig und nun zu einer einzelnen Gestalt geworden. Er tritt fast immer neben bāryā auf, dessen Oberhaupt er ist.

${ }^{11}$ Zār ist die wichtigste Erscheinung des äthiopischen und nordafrikanischen Volksglaubens. Das Wort ist im Amharischen bezeugt, nicht aber im Altäthiopischen, eine Tatsache, die für seinen afrikanischen Ursprung spricht. Daher dürfte der Zār ursprünglich Bestandteil der Religion der kuschitischen Stämme gewesen sein; von Äthiopien aus ging er zwischen der Mitte des 16. und Mitte des 18. Jh. ins Amharische über. In Äthiopien bleibt der Zār-Komplex eine Erscheinung am Rande des Christentums und des Islam, in der sich verschiedene Praktiken und Vorstellungen überlagert haben. Mit dem Wort Zār bezeichnet man sowohl die Geistergattung als auch die Krankheit und den Besessenen. Die Redewendung „die Krankheit des Zār“ (s. unten) bedeutet die Krankheit, die vom oder durch den Zār verursacht wird und entspricht der in Äthiopien verbreiteten Vorstellung, dass die meisten Krankheiten der Zārbesessenheit zuzuschreiben sind. Es handelt sich dabei nicht um Dämonen, sondern um Geistergestalten, die eine bedeutsame Rolle im Leben der Äthiopier spielen, die mit einer bestimmten Familie eng verbunden sind - sie sind auch vererbbar - und einen wichtigen Platz bei den Familiengeschäften einnehmen (ausführlicher s. B. BURTEA, Zwei äthiopische Zauberrollen, 30-33).

${ }^{12}$ Es ist die Bezeichnung eines alten Besessenheitskultes. Təgrətyā wird öfters im Zusammenhang mit Epilepsie gebracht (s. B. BURTEA, Zwei äthiopische Zauberrollen, 88).

${ }^{13}$ Bezeichnung einer Krankheit mit heftigen Schmerzen, s. auch B. BURTEA, Zwei äthiopische Zauberrollen, $37 \mathrm{f}$.

${ }^{14}$ Mitten im Wort wird die Spalte gewechselt. Es geht weiter in der rechten Spalte Z. 12. Qwərtəmāt bezeichnet ein Symptom, das durch starke Schmerzen charakterisiert wird, die mit dem Zustand des Zerkauens in Zusammenhang gebracht werden. Daher vielleicht die Neigung vieler Wissenschaftler, es mit Rheumatismus zu identifizieren.

${ }^{15}$ Wəg'āt ist die Bezeichnung für den Seitenstich, s. B. BURTEA, Zwei äthiopische Zauberrollen, 35f.

${ }^{16}$ Bezeichnung für Paralyse, vgl. F. E. DobBerahn, Fünf äthiopische Zauberrollen. Text, Übersetzung und Kommentar. Walldorf-Hessen 1976, 146.

${ }^{17}$ Mit budā sind die Schmiede gemeint, über die man den Verdacht äußerte, ihre Augen würden eine schädliche Zauberkraft besitzen, sie selber fräßen menschliches Fleisch und manchmal nähmen sie die Gestalt einer Hyäne an. Die Behandlung eines von budā besessenen Kranken vollzieht sich in zwei Phasen: die Entdeckung des budā, d. h. der Kranke gibt den budā-Namen preis, und die eigentliche Heilung, die oft aus einer Vertreibung des Dämons besteht (zu budā s. auch B. BURTEA, Zwei äthiopische Zauberrollen, 59-62).

${ }_{18}$ Tayāyāž ist ein Nomen agentis von dem amharischen Verb täyayaza „verbunden sein“ und bezeichnet im magischen Kontext einen Dämonen, der mit einem Menschen innig verbunden ist. Vgl. F. E. DobBERAHN, Fünf äthiopische Zauberrollen. Text, Übersetzung und Kommentar, $129 f$.

${ }^{19}$ Es folgt der sogenannte Fānu'el-Hymnus, ein häufiger Bestandteil der Zauberrollen. Der Gattung nach gehört dieser Hymnus der Salām-Dichtung an. Darunter versteht man mehrstrophige Hymnen an die Dreifaltigkeit oder an eine ihrer Personen, an Maria, einen der Erzengel oder eine(n) Heilige(n), die alle mit dem Wort Salām - d. h. Gruß, Heil (dir) - beginnen. In der apokryphen äthiopischen Literatur ist Fānu'el einer der vierundzwanzig Ältesten, die in der Apk 4, 10 erwähnt werden. Sein Name kommt auch im Henochbuch vor: 59, 6; 60, 9; 71, 9. 13. Auf Grund des Reims kann man dreiversige und vierversige Strophen des Hymnus unterscheiden. Die Übersetzung versucht diese Versstruktur wiederzugeben. Ausführlicher zum Fānu'el-Hymnus B. BuRTEA, Zwei äthiopische Zauberrollen (wie Anm. 8), 64-69. 
Heil dir, Vertreiber der Dämonen, Fānu'el, vom Angesicht des erhabenen Schöpfers, damit sie die Menschen mit dem verderblichen Wort nicht anklagen.

Denn du bist der Engel der Gnade.

Heil dir, vertreib die saytānāt von mir. O Herr,

Fānu'el, sei wachsam ${ }^{20}$, um zu helfen.

Denn bei mir ist das Wunder seiner Menschwerdung.

Heil dir Fānu'el, wirke ein schönes Wunder!

Mit der schwarzen Lanze soll man nicht meine Seite durchbohren, die man für mich schmieden ließ.

Heil dir, deinen Namen wollen wir jeden Morgen preisen!

Fānu'el, tue alles täglich

zum Helfen und zum Retten!

Heil! Dein Schwert ziehe gegen meinen Feind mastemā

gegen die Macht, die Mühsal verursacht.

Fānu'el, Engel von Rāmā.

Heil dir, Fānu'el, Petrus, Priester innen und außen.

Wenn ich jeden Tag siebzigmal siebenmal sündige, vergib mir, wie es bestimmt ist.

Heiligtum der Tora, in das du eingetreten bist, Māryām, unsere Mutter.

Im Schoß der Hanna nahmen wir Zuflucht.

Schicke ihn, den Ya'qob, dass er uns bewahre,

indem er mit dem Speer des Kreuzes den Satan durchbohre.

Heil dir, rette mich immer und verlasse mich nicht heute,

Fānū'el, wachsamer Engel des Friedens.

Denn durch den Starken wird der Schwache gerettet

und durch Heilung wird der Kranke gesund.

O, Gott des Fānū'el, bewahre sie und rette sie von der Krankheit des bāryā und legewon magāňňă $\bar{a}^{21}$ und guśəmt ${ }^{22}$, 'āyna səlāa $\bar{a}^{23}$, 'āyna magāňňă ${ }^{24}$, nagargāa ${ }^{25}$ und gurguho ${ }^{26}$, dədəq ${ }^{27}$ und ganena qətr ${ }^{28}$, fəlsat. Durch dieses dein Wort ${ }^{29}$ rette sie, deine Magd, Walatta Māryām.

${ }^{20}$ Erste Nahtstelle.

${ }^{21}$ Damit ist der Dämon der Türschwelle gemeint, der für starke Bauchschmerzen verantwortlich sein sollte, s. B. BurteA, Zwei äthiopische Zauberrollen, 84f.

${ }^{22}$ „Chronic pleurisy with cough“, S. STRELCYN, Catalogue of Ethiopian manuscripts of the Wellcome Institute of the History of Medicine in London. Bulletin of the School of Oriental and African Studies 35 (1973) 47.

${ }^{23}$ „Evil spirit which possesses s. o. and keeps him from getting anything favorable; unfortunate who is afflicted by the evil eye of others; reluctance (to do s.th.), abhorrence of doing s.th. (induced by a sorcerer); sloth“, T. L. KANE, Amharic-English Dictionary. Wiesbaden 1990, 1293.

${ }^{24}$ Wörtlich „das Auge des m.“, es wird geglaubt, dass ein Dämon mit seinem bösen Blick Unheil bewirken kann.

${ }^{25}$ Es scheint ein Sammelbegriff für mehrere Krankheitsbilder zu sein. Öfter wurde es mit Epilepsie wiedergegeben, s. F. E. DobBERAHn, Fünf äthiopische Zauberrollen, $154 \mathrm{f}$.

${ }^{26}$ „An illness of the throat (possibly tonsillitis)“, T. L. KANE, Amharic-English Dictionary, 1945.

${ }^{27}$ Möglicherweise die Bezeichnung für unerwartetes Unglück, s. B. BURTEA, Zwei äthiopische Zauberrollen, 89.

${ }^{28}$ Bezeichnung für den Mittagsdämon, s. B. BuRTEA, Zwei äthiopische Zauberrollen, 89f.

${ }^{29} \mathrm{Im}$ Original fehlerhafte Morphologie qāka für richtig qāləka „deine Stimme, dein Wort“. 
(Z. 42-137 linke Sp. / Z. 82-119 rechte Sp. ${ }^{30} /$ Z. 120-168 rechte Sp.)

Im Namen des Vaters und des Sohnes und des Heiligen Geistes, des einen Gottes.

Gebet des Heiligen Susənyos ${ }^{31}$ betreffend das Entfernen der Krankheit von den Kranken und den Jugendlichen. [Dieses nützt] auch dem Mann und der Frau, deren Kind leben wird. Sie soll [eine Zauberrolle] schreiben [lassen] und sie sich umhängen, dass es nützt mit der Hilfe Gottes des Erhabenen und Gepriesenen bis in die Ewigkeit. Amen.

Das Gebet und der Segen des Heiligen Susənyos. Die Hilfe Gottes möge schützen und heilen von der Krankheit des zār und des təgrida ${ }^{32}$ und des qurāňňă ${ }^{33}$ deine Magd, Walatta Māryām. Es war einmal ein Mann, dessen Name Susənyos war. Er heiratete eine Frau und zeugte ein männliches Kind. Und zu ihrem Erstgeborenen kam Wərzəly $\bar{a}^{34}$ und sie sagte zu seiner Frau: „(...) dieses dein Kind, und sie übergab sie [?] dieser Frau, und er [?] empfing es, während sie lächelte. Und sie [Wərzəlyā] küsste seinen Mund und blies auf seinen Hals, und er starb plötzlich. Und als sie [die Mutter] die Leiche sah, sah sie seinen Zustand [und] weinte heftig, wobei sie sprach: [?]

Und als der Heilige Susənyos das Weinen seiner Frau hörte ${ }^{35}$, antwortete er und sagte zu ihr: „Was macht dich, Frau, weinen?"

Sie sagte ihm: „Diese Frau. Und zwar es ist eine Frau gekommen, deren Name Wərzəlyā ist.“ Und sie sagte ihm: „Ich nahm [?] dieses dein Kind und ich übergab es, und sie blies auf seine Hand und seinen Hals [?] und sie hat mein Kind aufgenommen." Als er die Rede hörte, erschrak er und stieg herab von seinem Pferd und nahm einen Speer in seine rechte Hand und ging los, um sie zu suchen. Und er fing an [zu suchen] und fand er eine alte Frau, indem sie auf dem Weg saß. Und er fragte sie und sagte: „[Wo] ist Wərzəlyā gegangen?“ Und sie sagte ihm: „In den Garten, der vor dir liegt, ist sie gegangen.“

Und der Heilige Susənyos [ging] in den Garten und er fand Wərzəlyā, da sie unter einem Baum saß, während sie zahlreiche Dämonen umgaben. Und der Heilige Susənyos stieg von seinem Pferd herab und wandte sein Gesicht nach Osten und streckte seine Hände aus und fiel ${ }^{36}$ auf seine Knien nieder, betete und bat [Gott], indem er sagte:

„O unser Herr Jesus Christus, der Gott der Christen und König Israels, ich will Wərzəlyā töten, damit sie nicht mehr Kinder töte und damit sie sich ihnen nicht mehr nähere. Und nachdem ich sie getötet habe, wird sie in meine Hand fallen und ich werde Zeuge sein für die Deinige und für deinen heiligen Namen."

Und während er so betete, hörte er eine Stimme aus dem Himmel (...) Jesus (...):

„Siehe es ist dir die Macht Gottes gegeben, damit du Wərzəlyā packest und mit ihr tuest, was du willst."

Und als der Heilige Susənyos dies hörte ${ }^{37}$, freute er sich sehr und ritt auf seinem Pferd, wobei er seinen Speer in seine rechte Hand nahm, und ging zu ihr, wobei er ihre rechte Seite durchbohrte. Und sie sagte ihm:

\footnotetext{
${ }^{30}$ Nach der zweiten Nahtstelle werden drei Zeilen einspaltig geschrieben. Einfachheitshalber werden sie mit den Zeilen der rechten Spalte gezählt werden.

${ }^{31}$ Die Legende und das Gebet des Heiligen Susənyos gehören zu den verbreitetsten Texten in den äthiopischen Zauberrollen. Das Interesse an dieser Legende liegt im exemplarischen Kampf eines Heiligen gegen das Dämonische, das verantwortlich für bestimmte Krankheiten ist.

${ }^{32}$ Təgrida bezeichnet einen Dämon und die durch Besessenheit verursachte Krankheit, s. B. BurTEA, Zwei äthiopische Zauberrollen (wie Anm. 8), 88.

${ }^{33}$ Bezeichnet eine angeborene Krankheit, s. B. BuRTEA, Zwei äthiopische Zauberrollen, 55.

${ }^{34}$ Wərzəlyā ist die kindertötende Dämonin. Ihr Name ist auch in den koptischen Zaubertexten in mehreren Schreibvarianten bezeugt: Berzelia, Berselia, Aberselia (s. auch B. BURTEA, Zwei äthiopische Zauberrollen, 77f.).

${ }^{35}$ Im Original steht „,sie hörte“.

${ }^{36}$ Statt sagada steht nur wa-gada und „seine Hände“ wird wiederholt.

${ }^{37}$ Im Original steht dittographisch faqadka „du willst“ statt sam‘ā ,er hörte“, das durch andere Parallelstellen gesichert ist.
} 
„O mein Herr Jesus Christus ${ }^{38}$ ! Ich beschwöre dich bei den sieben Rangstufen der Erzengel: Mikā'el und Gabr'el, Surā'el und Kirubel, Urā'el und Rāfă'el, Anānyāl und Suryāl und Salātyāl. Diese sind diejenigen, die vor dem Angesicht des Pantokrators der Welt [stehen] " " Und wer auch immer [?] du hast meinen [?] ersten Sohn getötet und ferner du wirst die Gebärmutter nicht bluten lassen.

Gebet und Segen des Heiligen Susənyos: Und die Kraft und Hilfe Gottes mögen sie bewahren und retten vor der Krankheit des zār und des təgridā, des tabib ${ }^{40}$ und buda und 'əğ sab'41. Rette und bewahre deine Magd Walatta Māryām.

Im Namen des Vaters und des Sohnes und des Heiligen Geistes, des einen Gottes.

Gebet betreffend die Krankheit der Schwangerschaft: melāyo, malāləyos ${ }^{42}$, der nach Damaskus herabgestiegen ist, Jesus, der du die Kraft des Hagels und des Windes ${ }^{43}$ gerinnen lässest, in gleicher Weise lasse gerinnen, befestige ihr Menstruationsblut ${ }^{44}$ und öffne die Gebärmutter deiner Magd Walatta Māryām.

\section{(Z. 138-173 linke Sp.)}

'atam, katam, sarkatam ${ }^{45}$. Das Blut und das Wasser, das aus deiner Seite herauskam und floss, o unser Herr Jesus Christus, Schwert! Sieben Worte des Evangeliums! So wie Satana eingesperrt wurde, so auch sperre ihr Blut ein und nicht (...) ihr Fleisch und festige den Mutterleib im Bauch deiner Magd Walatta Māryām.

Sei es ein männliches Kind oder ein weibliches durch den Vater, seinen Namen Marā'el, für den Sohn, seinen Namen Mənātir, für den Heiligen Geist, seinen Namen 'abəyātir, qanātawi, satanāwi, satanāwi, qaranāwi, melossawi, tā'osāwi ${ }^{46}$, durch die Kraft dieser deiner [magischen] Namen, [du], der die Gebärmutter von Hannā und von Sarā geöffnet hast, so wie bei diesen, so auch öffne die Gebärmutter deiner Magd Walatta Māryām.

(Z. 173-184 linke Sp. / Z. 1-10)

Im Namen des Vaters und des Sohnes und des Heiligen Geistes, des einen Gottes.

Gebet betreffend die Festung des Kreuzes ${ }^{47}$ :

${ }^{38}$ In anderen Varianten steht an dieser Stelle Susənyos.

${ }^{39}$ Die Folge der Aussage Wərzəlyās - hier ausgelassen - lautet: ,... ich werde nicht mehr den Weg gehen, auf dem dein Name ist, und in die Häuser deiner Christen, die deines Namens gedenken und rezitieren die Schrift deines Kampfes und auch nicht zu dem, der diese Schrift trägt, sei es Mann oder Frau oder Kind oder Jüngling oder Greis, bis in alle Ewigkeit, Amen!“, zitiert nach F. E. DobBERAHN, Fünf äthiopische Zauberrollen (wie Anm. 16), 31, Z. 114-126.

${ }^{40}$ Es ist eine Bezeichnung für Zauberer.

${ }^{41}$ Der erste Teil ist amharisch, der zweite altäthiopisch und das Ganze bedeutet „,die Hand der Menschen“. Damit sind magische Handlungen von feindlichen Menschen gemeint, die bestimmte Krankheiten verursachen können.

${ }^{42}$ Es geht dabei um Varianten von melos, die Abänderungen des Namens Salomo darstellen und als Geheimnamen von Christus gelten, s. F. E. DobBerahn, Fünf äthiopische Zauberrollen, 144 und B. BurTEA, Zwei äthiopische Zauberrollen, 47ff. Salomo gilt als bedeutende Gestalt der Geschichte, die laut Apokryphenliteratur die Dämonen zu bezwingen vermag. Die bekanntesten magischen Werke dieser Gattung sind „Das Netz Salomons“ und „Der Spiegel Salomons", s. ebd., 5 Anm. 30f.

${ }^{43}$ Andere Varianten haben „,durch den Wind“, s. W. H. Worrell, Studien zum abessinischen Zauberwesen. Zeitschrift für Assyriologie 24 (1910) 59.

${ }^{44}$ Dahinter steht die Vorstellung, durch das Gerinnen des Blutes entstehe der Foetus.

${ }^{45}$ Es handelt sich um das „Gebet des Blutes“. Die magischen Worte enden auf -am um mit dam „Blut“ zu reimen.

${ }^{46}$ Es folgt eine Reihe magischer Namen Gottes hier als Dreifaltigkeit dargestellt. Der Glaube an die magische Wirkung des Namens ist das charakteristische Element der sogenannten Asmat-Gebete. Diese Texte bestehen aus Namenslisten verschiedenen Ursprungs (ägyptischen, griechischen, jüdischen, islamischen usw.), wie z. B. in dieser Liste tā'osāwi, das auf das griechische $\theta \varepsilon$ cós „Gott" zurückgeht.

${ }^{47}$ Der folgende magische Text „Festung des Kreuzes“ hasura masqal wird der Tradition nach dem Propheten Jeremias zugeschrieben, s. B. BuRTEA, Zwei äthiopische Zauberrollen (wie Anm. 8), 45 und Ders., Hasurä Mäsqäl. In: Encyclopaedia Aethiopica II (2004) [im Druck]. 
Kreuz, [unsere] Kraft ${ }^{48}$

Kreuz, unsere Macht

Kreuz, das Heil unserer Seele

Kreuz, unsere Erlösung

Kreuz, Besieger des Feindes

Kreuz, Unterwerfer des Feindes

Kreuz, Vernichter ${ }^{49}$ des Feindes

Kreuz, Zerbrecher des Feindes

Kreuz über jede Sache

Kreuz, Führer für die Blinden

Kreuz, Stab für die Schwachen

Kreuz, Prophezeiung der Propheten

Kreuz, Predigt der Apostel

Kreuz, durch dieses dein Wort sollen die furchtbaren Dämonen und die bösen Geister (...) fern gehalten und durch dieses dein Wort sollen fern gehalten (...) Feind.

Bewahre und rette sie, die Walatta Māryām, denn es gibt kein Ding, das Gott unmöglich ist.

\section{Die Illustrationen der Zauberrolle}

Während sich die Wissenschaft mit den Texten der Zauberrollen bereits eingehend beschäftigt hat, sind die beigefügten bildlichen Darstellungen bislang wenig untersucht worden. Daher sind viele der Probleme, die die Funktion und Stellung der Zauberrollenillustrationen innerhalb der äthiopischen Ikonographie betreffen, noch nicht eindeutig gelöst. Die bildende Kunst, insbesondere die Kirchenmalerei und die Buchillustration, stand in Äthiopien in sakralem Kontext. Historisch bildete sich die sakrale Malerei in dem Maße aus, wie sich die politische und religiöse Macht im 14. und insbesondere im 15. Jahrhundert unter der Wirkung des Königs Zar'a Ya'qob (1434-1468) zentralisierte. Die Malerei dieser Jahrhunderte dient ausschließlich religiösen Zwecken. Die Darstellungen sind schematisiert und weisen keine perspektivischen Verkürzungen auf.

In der Gondarzeit im 17. Jh. griff eine realistischere Auffassung und eine Vorliebe für kräftige Farben Platz. In der Folgezeit erhält dieser Stil eine dekorative und erzählende Ausprägung $^{50}$. Demgegenüber steht der sogenannte geometrische Stil, der lokal in den Klöstern gepflegt wurde. Die Illustrationen der Zauberrollen scheinen auf diesen geometrischen Stil auch Schoa-Stil aus dem 18. und 19. Jh. genannt - zurückzugehen ${ }^{51}$, wie aus der Darstellung von Augen und Gesichtern zu schließen ist. Die Dabtarā bedienen sich beider erwähnten Traditionen, wenn sie auch den geometrischen Stil bevorzugen ${ }^{52}$, ferner der komplizierten Flechtwerkmotive $^{53}$. Letztes ist vermutlich der bäuerlichen Tradition geschuldet, da Bauern, wie bereits erwähnt, die Hauptkunden für Zauberrollen waren.

\footnotetext{
${ }^{48}$ Das Pronominalsuffix -na wurde ausgelassen.

${ }^{49}$ Drittes Bild: Schutzengel. Der übrige Text ist einspaltig und wird getrennt gezählt.

${ }^{50}$ Die bedeutsamen Phasen der äthiopischen Malerei sind von JÄGER beschrieben, O. JÄGER, Äthiopische Zauberrollen und ihre Bilder. Baeseler Archiv N. F. 14 (1966) 150ff. und E. HAMMERSCHMIDT - O. A. JÄGER, Illuminierte äthiopische Handschriften (Verzeichnis der orientalischen Handschriften in Deutschland XV). Wiesbaden 1968, 7-15.

${ }^{51}$ Vgl. S. ChOJNacki, Major Themes in Ethiopian Painting. Wiesbaden 1983, 494ff.

${ }^{52} \mathrm{Vgl}$. die Abbildungen bei J. MerCiER, Les plus anciens rouleaux protecteurs éthiopiens de la Bibliothèque Nationale de Paris. Annales d'Éthiopie 10 (1976) Tafel XLII, Abb. a und c, Tafel XLIII, Abb. a.

${ }_{53}$ J. Mercier, Zauberrollen aus Äthiopien. München 1979, 28. Die häufigen Engelsgestalten sind der ersten Periode zuzuordnen.
} 
Im Unterschied zur sakralen Malerei, die der Erbauung des Gläubigen diente, richten sich die magischen Bilder an einen durch einen Dämon besessenen Kranken. Sie sollen direkt auf den entsprechenden Geist (zār, bāryā usw.) einwirken ${ }^{54}$.

Die Dabtarā teilen diese Zauberrollenbilder im Allgemeinen in zwei Kategorien ein: śə‘’’l abbildende Bilder, d. h. Bilder, die darstellen, was sichtbar ist und die die Geschichte einer Person zum Thema haben ${ }^{55}$, und tälsäm, die Talismane, d. h. die magischen Zeichen, die wirkmächtigen Bilder $^{56}$. Das Wort tälsäm kommt aus dem arabischen tilasm, das seinerseits aus dem griechischen $\tau \varepsilon ́ \lambda \varepsilon \sigma \mu \alpha$ „Tribut, Steuer“, aber auch „wirkmächtiger Gegenstand“, abgeleitet ist ${ }^{57}$. Für die Dabtarā bedeuten die tälsäm, „la haie qui défend contre l'oeil de l'ombre, le mauvais oeil““58.

Weitgehend unklar ist die Beziehung zwischen Bildern und Texten. Ein Zusammenhang wird von den meisten Äthiopienspezialisten vermutet, lässt sich aber kaum nachweisen. J. MERCIER vertritt eine, wie mir scheint, plausible Meinung, wonach es dabei nicht um die bildliche Wiedergabe der Texte geht, sondern um einen Parallelismus: d. h. die Bilder vermitteln ihre eigenen Aussagen, welche diejenigen der Texte ergänzen: „Le regard est dans les tälsäm ce que la parole est dans les asmat “59 und „Ce qui compte pour les magiciens interogés c'est leur fonction [der Bilder, B. B.] curative qui consiste à faire cesser le travail de tel ou tel démon ... Il faut, par conséquent, renoncer au principe scolaire de la signification universelle impliquant que les peintures sont conçues comme des illustrations de textes, au profit du dévoilement de l'étroite relation existant entre la situation d'usage et la stylistique des peintures ${ }^{\text {“60 }}$. So sind die Bilder Bestandteil des gesamten Heilungsprozesses, dem sich der Patient unterzieht ${ }^{61}$.

Charakteristisch für die äthiopischen Zauberrollen sind die meist drei, seltener vier Bilder, die den magischen Text begleiten. Das ikonographische Programm der hier untersuchten Rolle besteht aus vier Illustrationen.

Das erste Bild stellt einen Schutzengel dar, der als Einleitungsmotiv einer Zauberrolle sehr oft anzutreffen ist. Das ganze Bild ist $34 \mathrm{~cm}$ lang, wenn man die Flechtwerkmotive, die das eigentliche Bild einrahmen, dazu rechnet. Das Bild des Engels ist sehr einfach und weist die typischen Merkmale dieser Art von Darstellungen auf: ein drohendes Schwert in der rechten, die Schwertscheide in der linken Hand, die frontale Position des Gesichts mit den großen Augen, die großen Flügel, die die Gestalt links und rechts einrahmen. Der Stil der Zeichnung ist schlicht, fast geometrisch, die Farben sind sehr blass und beschränken sich auf schwarz, gelb und rot. Auffällig sind dabei die fast menschlichen Züge des Engelsgesichts mit den schwarzen Haaren und Augenbrauen sowie mit der stark konturierten Nase und die orientalische Kleidung bauschige Hosen und Schuhe mit gebogenen Spitzen. Der Mund ist wie bei vielen Engeldarstellungen nicht gezeichnet. Der Gedanke, dass jeder Mensch einen Schutzengel hat, wie es in der abendländischen und koptischen Angelologie der Fall ist ${ }^{62}$, spielt auch in Äthiopien eine

\footnotetext{
${ }^{54} \mathrm{Vgl}$. J. Mercier, Sur un type particulier de talisman et les enseignements que l'on peut en tirer à propos de l'art magique. Documents pour servir à l'histoire de la civilisation éthiopienne 6 (1975) 110.

${ }_{55}^{5}$ Sa'al ist der Terminus für die allgemeine religiöse Malerei, J. Mercier, Sur un type particulier de talisman et les enseignements que l'on peut en tirer à propos de l'art magique, 103.

${ }^{56} \mathrm{~J}$. MercIER, Zauberrollen aus Äthiopien, 24. Für die verschiedenen Arten von tälsäm vgl. J. MERCIER, Sur un type particulier, 103. Für die Interpretation der äthiopischen Talismane siehe J. MERCIER, Art that heals (wie Anm. 6), $57 \mathrm{ff}$.

${ }^{57}$ Vgl. W. LeSLau, Arabic Loanwords in Ethiopian Semitic. Wiesbaden 1990, 79 und E. DoutTÉ, Magie et religion dans l'Afrique du Nord. Alger 1908, 143.

${ }^{58}$ D. LifChitZ, Amulettes éthiopiennes. Minotaure 2 (1933) 72.

${ }^{59} \mathrm{~J}$. Mercier, Les plus anciens rouleaux protecteurs éthiopiens de la Bibliothèque Nationale de Paris, 233. Vgl. auch Dens., Sur un type particulier, 111: „Le talisman est à la peinture religieuse ce que l'incantation est au récit.“

${ }^{60} \mathrm{~J}$. Mercier, Les plus anciens rouleaux protecteurs éthiopiens, 232.

${ }^{61} \mathrm{~J}$. Mercier, Les däbtära et le vocabulaire de la magie et de la médecine. Documents pour servir à l'histoire de la civilisation éthiopienne 7 (1976) 113.

${ }^{62}$ E. WAGNER, Die Illustrationen der äthiopischen Zauberrollen der Sammlung Littmann. In: W. HoENERBACH (Hg.), Der Orient in der Forschung. Festschrift für Otto Spies zum 5. April 1966. Wiesbaden 1967, 719.
} 
Rolle $^{63}$. Die Frage, ob hier ein privater oder ein allgemeiner Schutzengel gemeint ist, lässt sich nicht eindeutig beantworten. Für den allgemeinen Schutzengel würde die Existenz eines Hymnus für den mal'aka 'uqābe (wörtlich Schutzengel) sprechen. Dieser Hymnus berichtet unter anderem: „Heil dir, o Schutzengel, der du in der ganzen Welt Beschützer dessen bist, der im vollkommenen Glauben auf dich vertraut ${ }^{64}$ !" E. WAGNER führt aber auch einige Indizien an, die dem Erzengel Fānu'el diese schützende Rolle zuschreiben: er soll sein Schwert gegen den Feind Mastema ziehen (vgl. die Übersetzung oben), oder, wie eine Hymnus-Stelle beschreibt, er soll schützen (hier nämlich sie, die Rollenbesitzerin) ${ }^{65}$.

Das nächste Bild ist viel kleiner - 2,5 cm Höhe und 4,5 Breite - und befindet sich in der rechten Spalte des Texts. Das Bild gehört zur Kategorie der tälsäm, der magischen Bilder. Ihre besondere Wirkung liegt darin, dass sie ohne jegliche Vermittlung auf die Dämonen einwirken sollen $^{66}$. Dieses Bild besteht aus zwei Elementen. Das erste, das wie ein Rad aussieht, könnte eine sehr vereinfachte Darstellung eines achtstrahligen Sterns sein, eines Motivs, das sich häufig bei den Illustrationen der magischen Rollen findet. Das andere Element dieses Bildes ist ein Kreuz. Die Tatsache, dass das Kreuz als Abwehrmittel gegen die schädliche Wirkung der Dämonen eingesetzt wird, betont seine Bedeutung im religiösen Leben der christlichen Äthiopier. Eine erste historische Erwähnung ist aus der Kirchenordnung des Königs Zar'a Ya'qob - hier in der Paraphrase A. Dillmanns - zu gewinnen: „Ebenso befehle er, daß alle christlichen Leute Äthiopiens an ihren Kleidern, Gürteln, Stäben, Waffen, Pflugscharen und allen Acker- und Arbeitgeräthen, ebenso die Weiber an ihren Handarbeiten das Kreuzeszeichen machen, denn das Kreuzeszeichen sei eine Pein für den Satan, wie der Senodos sage ${ }^{\text {"67 }}$. Die abwehrende Funktion in der Magie wird nicht nur durch Zeichnungen, sondern auch durch Texte wie die „Festung des Kreuzes“ (s. Übersetzung) oder wie der folgende realisiert: „Auf das Holz deines einzigen Kreuzes stütze ich mich. Denn meine Kraft und meine Zuflucht ist das Kreuz. Wer im Schatten des Kreuzes sitzt, Gott des Himmels ist seine Rettung"68.

Das dritte Bild stellt wiederum einen Engel dar. Die Länge des Bildes samt der einfachen Flechtwerkmotive oben und unten beträgt ca. $15 \mathrm{~cm}$. Die Darstellung ist sehr schlicht. Der Kopf ist von einem Nimbus und von Flügeln umgeben. Wie der andere Engel, so ist auch dieser großäugig und mit großer Nase dargestellt, die Stelle des Mundes ist ausgespart. Dieser Engel trägt kein Schwert in den sehr schematisch aufgefassten Händen. Die Füße sind nicht zu sehen.

Das Ende des Pergamentstreifens ist sehr angedunkelt. Dennoch könnte man an dieser Stelle ein viertes, abschließendes Bild erahnen. Alles, was man dabei erkennen kann, ist ein Gesicht mit Nimbus und die Konturen von Engelsflügeln. Möglicherweise handelt es sich hier wiederum um einen Schutzengel.

${ }^{63}$ Die Vorstellung von den schützenden Geistern wird auch von den Ethnologen dokumentiert. H. S. LEWIS, Spirit Possession in Ethiopia: an Essay in Interpretation. In: S. Segert - A. J. E. Bodrogligeti (Hg.), Ethiopian Studies Dedicated to Wolf Leslau. Wiesbaden 1983, 469f. redet von einem wuk'abi als ,such a guardian spirit may protect an individual, or a family, lineage, a community“; M. LEIRIs, La croyance aux génies «zar» en Éthiopie du Nord. Journal de psychologie normale et pathologique 35 (1938) 123, bezeichnet den waqabi als ,sorte de génie personnel ou d'ange gardien“. Er fügt hinzu „Mais cette notion même de waqabi ne peut, du point de vue européen, se définir clairement; en même temps qu'elle implique l'idée d'esprit gardien, elle implique celles de chance, de charme, de beauté, de correction morale, de prestige ... “

${ }^{64}$ Text und Übersetzung bei E. WAGNER, Die Illustrationen der äthiopischen Zauberrollen der Sammlung Littmann, 719 Anm. $76 \mathrm{ff}$.

${ }^{65}$ Ebd., $722 \mathrm{f}$.

${ }^{66}$ J. Mercier, Sur un type particulier (wie Anm. 54), 113 und Ders, Art that heals, 99, wo er ihre Wirkung charakterisiert: „Refusing the demon access to the body, the talisman is an interdiction.“

${ }^{67}$ A. Dillmann, Über die Regierung, insbesondere die Kirchenordung des Königs Zar'a-Jacob. In: Abhandlungen der Königlichen Akademie der Wissenschaften zu Berlin, phil.-hist. Klasse. Berlin 1884, 65. Über Kreuz als Heilmittel siehe auch J. Mercier, Art that heals (wie Anm. 6), 64-71.

${ }^{68}$ Der Zauberrollentext bei B. BurTEA, Zwei äthiopische Zauberrollen (wie Anm. 8), 20, Z. 19-22. 
Abschließend könnte man festhalten, dass die Zauberrollen wichtige Zeugnisse der Verbreitung und Pflege des Altäthiopischen während der letzten Jahrhunderte sind. Trotz Nachlässigkeiten ist die Sprache der Zauberrollen sowohl in der Morphologie als auch in der Syntax mit dem „klassischen“ Gə‘ $ə z$ identisch. Die Zauberrollen zeugen zugleich vom Gebrauch des Altäthiopischen außerhalb des Kirchenlebens. Sowohl die Sprache der Zauberrollen als auch die Bilder belegen die Kontinuität der überlieferten Tradition.

Die Zauberrollen geben außerdem Auskunft über die Intensität der Verbreitung des Christentums unter der breiten Masse der Äthiopier. Sie zeigen, wie vertraut die Schreiber der Amulette mit den Schriften des Alten bzw. des Neuen Testaments waren, aber auch die synkretistischen Entwicklungen innerhalb des äthiopischen Christentums.

\section{Summary}

An Ethiopian magic scroll and its images

In its introduction this article presents the historical and social background of Ethiopian magic and of its well-known objects, the magic scrolls. They are prepared by the dabtara - not ordained clerics of the Ethiopian church - for a person suffering from troubles.

The magic scroll written for a woman named Walatta Māryām (the daughter of Mary) was made using three bands of parchment of equal width sewn end to end. The text comprises wellknown magical prayers and incantations like the hymn of the angel Fānū'el, the legend of the Saint Susənyos or the Rampart of the Cross. The text is accompanied by four images. Three of them represent a protective angel. The other one is a tälsäm, a magical drawing, with the sign of the cross. 